\title{
Glasdegib Maleate
}

National Cancer Institute

\section{Source}

National Cancer Institute. Glasdegib Maleate. NCI Thesaurus. Code C156894.

The maleate salt form of glasdegib, an orally bioavailable small-molecule, smoothened (SMO) receptor inhibitor, with potential antineoplastic activity. Upon oral administration, glasdegib targets, binds to and inhibits the activity of SMO. This inhibits the activity of the Hedgehog $(\mathrm{Hh})$ signaling pathway and inhibits the growth of tumor cells in which this pathway is aberrantly activated. SMO, a transmembrane protein, is involved in $\mathrm{Hh}$ signal transduction. The Hh signaling pathway plays an important role in cellular growth, differentiation, repair, and cancer stem cell (CSC) survival. Constitutive activation of $\mathrm{Hh}$ pathway signaling has been observed in various types of malignancies and is associated with uncontrolled cellular proliferation in a variety of cancers. 